\title{
Field-Based Waveguide Filter Synthesis in the Time Domain
}

\author{
Aurélie Sallier, Jens Bornemann, and Wolfgang J.R. Hoefer \\ Dedicated to Professor Peter Russer on the occasion of his 60th birthday
}

\begin{abstract}
Computer modeling of components and systems has revolutionized engineering design. Yet, most computer-aided design (CAD) approaches follow the strategy employed in traditional practice: an initial model, created with empirical or approximate rules, is tested (analyzed) and modified (optimized) until it meets specifications. The key concept in this CAD approach is thus the efficient combination of numerical analysis with an optimization strategy. In this paper we explore a new approach to electromagnetic design of waveguide filters and aim at creating an alternative design methodology. The main idea consists in exploiting the intrinsic optimization capability of electromagnetic fields to minimize energy storage and losses, not by means of a variational formulation, but by exploiting the topology of three-dimensional standing wave pattern created in space. This new field synthesis approach relies on time-domain modeling of electromagnetic fields and employs the Transmission-Line Matrix (TLM) modeling method. After a theoretical introduction to the synthesis methodology we describe a step-by-step synthesis of an iris-coupled rectangular waveguide bandpass filter and verify the result by performing a rigorous field analysis of the synthesized topology.
\end{abstract}

Keywords: Field synthesis, Waveguide filters, Time domain modeling, Optimal design

\section{Introduction and background}

Traditional Computer-Aided Design (CAD) in microwave and millimeter-wave engineering based on analysis with an optimization strategy has been very successful for two reasons:

a) Field analysis of passive structures yields unique results, while direct field synthesis is usually not unique;

b) Optimization theory and techniques have become highly sophisticated, powerful and robust.

CAD of electromagnetic wave structures with dimensions of the order of the spatial wavelength (microwave, millimeter-wave, photonic, and high-speed digital components and circuits), relies either on direct electromagnetic analysis, or on the analysis of surrogate models (empirical formulae, equivalent circuits, neural networks), combined

Received ||||||||||||||| . Revised December 2, 2002.

A. Sallier, Electronics and Communications Systems, INSA, 35043 Rennes, France.

J. Bornemann, W.J.R. Hoefer, Department of Electrical and Computer Engineering, University of Victoria, Victoria, BC, Canada V8W 3P6.

Correspondence to: Wolfgang J.R. Hoefer.

E-mail: whoefer@ece.uvic.ca with optimization. The relationship between the fine field model and the coarse surrogate model is often referred to as Space Mapping. A number of commercial field-based and circuit-based CAD tools embody this analysis-andoptimization paradigm.

In this paper we explore a new approach to electromagnetic design of wave structures and describe an alternative design methodology applied to waveguide bandpass filters. We take advantage of the inherent optimization property of electromagnetic fields to minimize energy storage and losses, not by means of a variational formulation, but by exploiting the topology of three-dimensional standing wave pattern created in space. This new approach relies on time-domain modeling of electromagnetic fields by means of the Transmission-Line Matrix (TLM) modeling method.

The following simple 1D example conveys the essence of this new approach: design (synthesize) a one-dimensional transmission line resonator by injecting the desired resonant frequency from a matched source into a shortcircuited transmission line. The resulting standing wave pattern yields the required boundary position exactly and without optimization. In fact, it minimizes the energy stored in the structure at the resonant frequency. This approach works even if the transmission line impedance and propagation constant are inhomogeneous, dispersive, and nonlinear. It may be implemented experimentally or as a numerical procedure, and is a true field-based synthesis by monochromatic injection.

We have already demonstrated that this approach can be extended to 2D and 3D structures by synthesizing cavities, waveguide discontinuities and simple filter structures [1-4]. However, in this paper we propose a systematic design methodology for electromagnetic wave structures and apply it to waveguide bandpass filter synthesis.

Given the success and the high level of maturity of CAD by analysis and optimization, one might ask why an alternative approach would be necessary, and what would be its advantages. Firstly, optimization in the context of CAD usually means minimization of the discrepancy between actual and desired circuit responses, which does not automatically imply optimization in the sense of minimizing losses and sensitivity to tolerances. Secondly, many optimizers treat the analysis engine as a "black box" and establish phenomenological rather than physical relationships between the parameters of a component and its functionality. Thirdly, many optimization strategies, such as random optimizers and genetic algorithms, may require a large number of analysis cycles, 
resulting in large computational demands. Space Mapping, statistical design and adjoint sensitivity concepts have successfully mitigated these issues. However, field synthesis does not require extrinsic optimization since the field itself has the necessary intrinsic optimization properties, properties that are not exploited in the analysis phase. Designers will thus be able to synthesize, with minimum numerical effort, physically optimal structures yielding the desired (specified) response. Field synthesis will be to wave structure design what circuit synthesis is to circuit design. On a final note, since field synthesis employs the same field model as analysis, it is fully compatible with analysis-and-optimization techniques so that it will complement them rather than compete with them. In fact, it will be possible to use field synthesis to generate starting values for an optimization process when they cannot be generated by other means. Thus, we expect future commercial field-based design tools for wave structures to contain a field synthesis option in addition to conventional optimization features.

\section{Methodology and general field synthesis approach}

The electromagnetic field has the following inherent optimizing properties:

1. It can minimize stored energy;

2. It can minimize dissipated energy (losses);

3. It can minimize sensitivity to boundary variations and tolerances.

These properties have been used extensively in electromagnetic analysis, for example in variational formulations of field problems and in the finite element method (FEM). Our goal is to exploit this intrinsic optimization capability of the electromagnetic field to directly synthesize the optimal structure dimensions.

Figure 1 compares the classical optimization approach with the new paradigm proposed in this paper. Note that

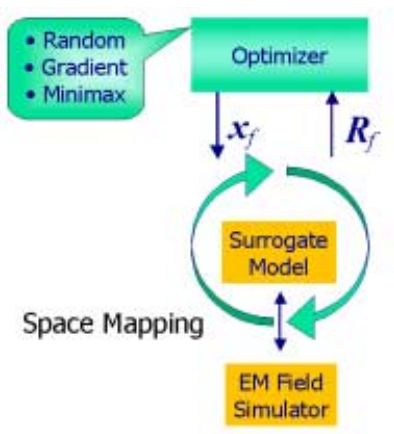

(a)

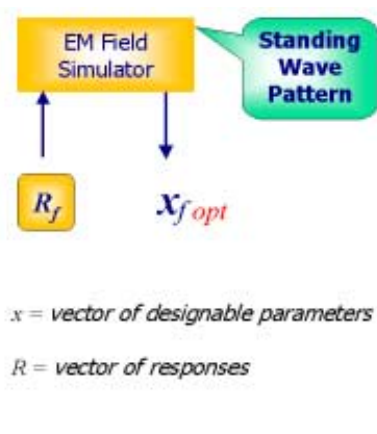

(b)
Fig. 1. (a) Classical optimization using space mapping, and (b) Direct field synthesis proposed in this paper. the synthesis involves the injection of the vector of desired responses (specifications) into the field simulator, and the output of optimal designable parameters by means of multi-dimensional standing wave patterns. In this paper, designable parameters are the dimensions and shapes of boundaries of a waveguide filter.

The filter synthesis procedure by means of a TLM field simulator can be summarized as follows:

1) Create an equivalent circuit consisting of lumped elements and 1D transmission line elements that satisfies the desired specifications;

2) Generate a starting geometry (first guess);

3) Identify the designable boundary parts;

4) From specifications and the equivalent circuit, determine a number of characteristic frequencies equal to the number of designable parameters of the structure;

5) In the actual synthesis phase, define an appropriate resonant sub-structure, discretize it into a TLM network, and replace the designable boundary parts by matched TLM sources that inject sampled monochromatic signals at the characteristic frequencies;

6) Extract the synthesized boundary geometry from the envelope of the resulting standing wave pattern;

7) Verify the performance of the design by means of a final analysis; if necessary, repeat the synthesis procedure by using the previously obtained structure as a starting geometry.

In the following, each step will be described in detail. In section 3 of this paper, we will elaborate on each step while synthesizing a two-resonator waveguide bandpass filter.

\subsection{Creation of a suitable equivalent circuit}

The synthesis of equivalent lumped element filters is a classical problem and has been described extensively in many textbooks [5]. Although a variety of circuit representations of RF/microwave/millimeter-wave and optical components exist in the open literature, the synthesis of circuit element values to satisfy given component specifications is often cumbersome. A recently introduced method [6] will facilitate this process. Its advantages include a strictly enforceable topology without the need for similarity transforms; the possibility of synthesizing both symmetric and asymmetric characteristics; no restrictions on the number or type of equivalent-circuit elements; the possibility to constrain specific elements in sign and value; and the avoidance of round-off errors which usually plague extraction methods.

An alternative approach is to use a circuit simulator with optimization to design a circuit consisting of lumped elements and transmission line elements that satisfies given specifications. The equivalent circuit enables us to identify the characteristic frequencies associated with the designable parameters. It is desirable that the structure of the equivalent circuit closely emulates the structure of the actual waveguide filter so that 


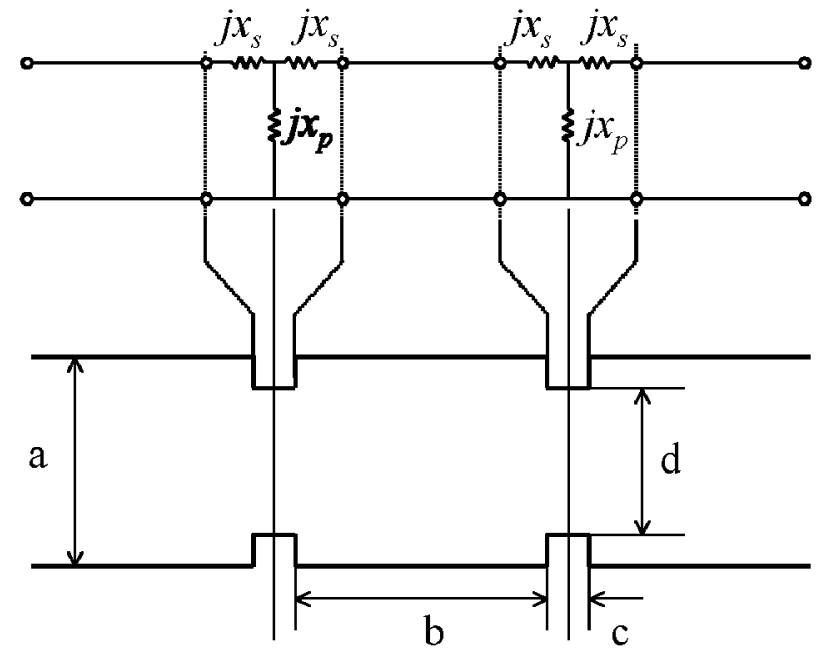

Fig. 2. A simple waveguide bandpass filter and its natural equivalent circuit consisting of lumped elements and sections of transmission line.

each circuit element can be associated with a geometrical feature of the structure, as shown in Figure 2. In this example, the septa of the waveguide filter are represented by a T-network of inductances, while the resonator and input/output sections are transmission line sections. Strictly speaking, such equivalent circuits are correct representations of the real microwave components only at one frequency, but the frequency dependence of the equivalent circuit parameters is usually a very smooth function so that it is easy to extend the validity of the circuit model to a sufficiently wide band around the design frequency. In any case, the equivalent circuit of a component derived using network synthesis can usually be transformed into a physically realistic circuit such as shown in Figure 2.

Note that potential higher-order mode interactions between the septa will be accounted for in the field synthesis phase.

\subsection{Generation of a starting geometry}

It is well known that many microwave circuit functions can be realized by a number of possible geometries, meaning that the synthesis process is not unique. For example, a waveguide bandpass filter can be realized by means of either irises or posts of various cross-sections. It is thus important to select a starting geometry that uniquely determines the desired type of filter geometry, and to predict its approximate dimensions (first guess geometry). Marcuvitz Waveguide Handbook [7] is a classical resource for waveguide component design; it contains a wide range of discontinuities, their equivalent lumped element circuits, and both formulas and design charts relating the element values to the discontinuity dimensions. Similar information can be found in a number of popular microwave handbooks and texts.
The synthesis is launched from the starting geometry. In the unlikely event that the starting geometry exactly realizes the desired specifications, the field synthesis will not yield any changes but will confirm the existing dimensions. However, if the starting dimensions are different from, but close to, the optimal values, the synthesis yields a topology that resembles the first guess design. Hence, if the synthesis starts from an iris-type topology, the result will be an iris filter. In other words, the choice of the first guess narrows the range of possible solutions by introducing specific constraints. Furthermore, the selected topology must be feasible, since otherwise the synthesis procedure cannot converge. All these initial considerations apply to traditional synthesis by analysis and optimization as well, however, the synthesis procedure provides more geometrical flexibility in the realization of the design goal. This is illustrated in Figure 3 which compares typical results of analysis with optimization (a) and of field synthesis by monochromatic injection (b). In the case (a) the penetration of the septum into the waveguide has been modified without changing its rectangular shape. In the case (b), the synthesis yields an optimal topology that not only gives the desired S-parameters but also minimizes losses and sensitivity to tolerances. As mentioned before, if the first guess is already optimal, the contour will not be changed by the synthesis.

\subsection{Identification of the designable boundary parts}

Once the generic topology of the electromagnetic structure has been determined, it is necessary to specify which of its geometrical dimensions should be modified to yield the desired characteristics. (While the S-parameters of a waveguide filter could also be modified by changing the constitutive parameters of the materials it contains, we will focus exclusively on the geometrical dimensions as designable parameters in this paper). As we shall demonstrate later, the designable boundary parts will be replaced by sources that inject monochromatic fields into the structure at specific characteristic frequencies. The goal of the synthesis is to determine the shape and position of these boundary parts such that the structure has the same S-parameters as the specified equivalent circuit.

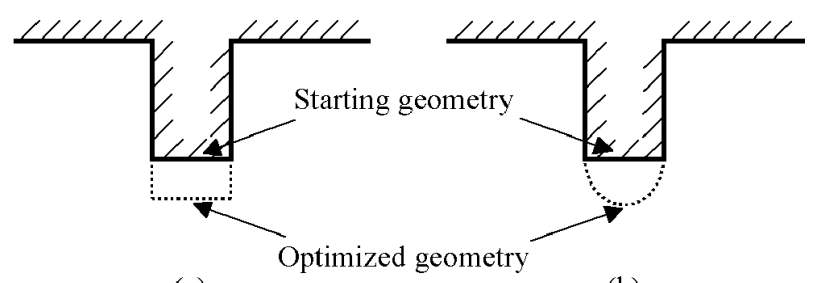

(a)

(b)

Fig. 3. Typical results obtained when optimizing the penetration of a septum into a waveguide. The starting geometry is identical in both cases. The optimized contour is dotted. (a) Result of analysis with optimization, (b) Result of monochromatic field synthesis. 


\subsection{Determination of the characteristic frequencies}

Characteristic frequencies are resonant frequencies of the structure itself, or of a subsection of the structure, that depend on one of the designable boundary parts. In the simple case of a 1D resonator, the characteristic frequency is its dominant resonant frequency which depends on the resonator length [4]. In a resonator with more complex boundaries, characteristic frequencies could be the resonant frequencies of different modes that could be selected such that each designable boundary part essentially controls a different resonant mode. In a partially open structure such as a filter, resonances must be created by strategically inserting electric or magnetic walls, which appear in the equivalent circuit as open or short circuits [2]. The resonant frequencies of the so created sub-circuits are the characteristic frequencies of the structure and can be determined directly from the values of the circuit elements.

\subsection{Monochromatic field injection}

Once the characteristic frequencies and the associated designable boundary parts have been identified, the actual synthesis phase begins. The first-guess structure (including the strategically placed electric or magnetic walls) is discretized into a 3D-TLM network of transmission lines, and the associated resonant mode is excited. Since the dimensions of the first-guess structure are usually not optimized, the resonant frequency will be different from the characteristic frequency determined from the equivalent circuit. However, the field distribution along the boundaries will be approximately the same as in the optimal configuration. The field distribution along the designable boundary part will be recorded and stored. Then, this boundary part is replaced by an array of matched sources, one for each of the TLM lines ending on that boundary, and a monochromatic sine wave at the characteristic frequency is injected with the amplitude and phase distribution recorded during the previous step. The resulting standing wave pattern reaches a steady state due to the losses introduced by the matched TLM sources, and the node line of the electric field represents the optimal contour of the designable boundary part.

This process is repeated for each of the designable boundary parts and usually converges after one step. However, the process can be repeated if necessary, for example if the change in one boundary part has a significant influence on the optimal position of another.

\subsection{Extraction of boundary geometry from standing wave pattern}

If the first-guess geometry is very close to the optimal geometry (within the mesh size of the TLM model), the standing wave pattern can be contaminated by the near field of the sources, particularly in the vicinity of an edge. To eliminate this effect, the node line is identified by extrapolating the standing wave field pattern to zero. Note that the resolution of this procedure is better than the TLM discretization since the standing field pattern has a smooth, well-defined envelope that can be extrapolated to zero with high accuracy even if the sampling points are located several cells from the node line [2].

\subsection{Verification and validation of design}

The results of the synthesis are verified and validated by a subsequent analysis of the synthesized structure. Either TLM or another numerical technique may be used. Experimental verification is always recommended, provided that the optimized topology can be realized within adequate mechanical and electrical tolerances, and the measurement system is carefully calibrated.

\section{Synthesis example - A waveguide bandpass filter}

We will now synthesize a simple waveguide bandpass filter in order to illustrate the methodology and the different steps described above rather than demonstrate the ultimate potential of the method. To ensure feasibility of our design, and for the sake of convenience and ready verification of the synthesis procedure, we begin with an actual waveguide filter, analyze it to get its $S$-parameters and equivalent circuit, and then use these as specifications for the synthesis. We expect the synthesized geometry to be different from the original structure, but it should have the same S-parameters.

Figure 4 shows the topology and the dimensions of a waveguide filter which can be modeled in $2 \mathrm{D}$ since the fields are uniform in z-direction. The dimensions have been arbitrarily selected, the boundaries are perfect electrical conductors, and the dielectric is air. We can find the

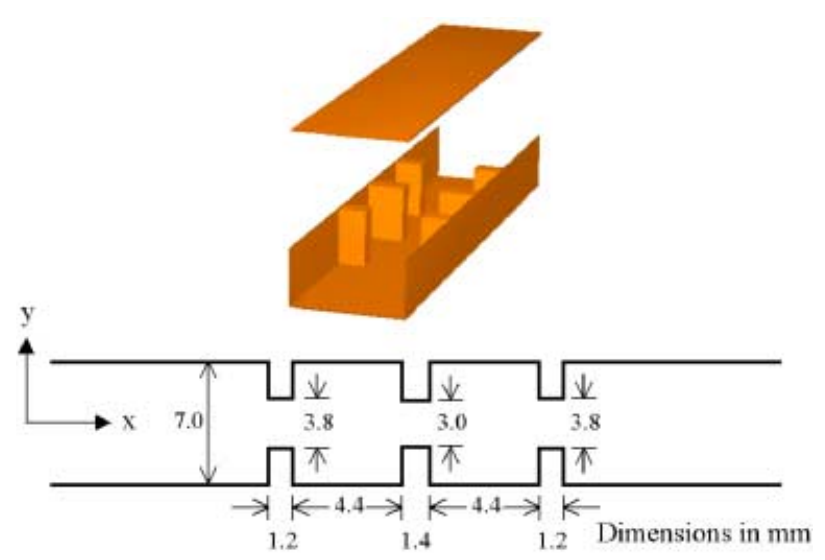

Fig. 4. Three dimensional view and dimensions of the waveguide bandpass filter which consists of two resonators formed by thick metallic irises. The structure is uniform in z-direction and can thus be modeled in 2D. The material inside the filter is air. (Proposed by W. Menzel). 
S-parameters of this filter as well as a suitable equivalent circuit by analyzing the structure with a numerical method such as TLM.

\subsection{Specification by equivalent circuit}

Figure 5 shows two possible equivalent circuits for an inductive iris in a waveguide (see, for example, Marcuvitz [7]). We can thus build an equivalent circuit for the entire filter by connecting such T-networks by means of transmission line sections as shown in Figure 6. The behavior of the equivalent circuit will be most realistic if we assume that the connecting transmission line sections have the dispersive characteristic impedance and phase velocity of the rectangular waveguide. (This does not include higher-order mode interaction between irises). However, at mid-band frequency, a TEM line section with the impedance and phase constant of the waveguide at that frequency will be a suitable approximation. The S-parameters of both the filter and its equivalent circuit are shown in Figure 7. Indeed, the equivalent circuit with dispersive transmission line sections is an excellent representation of the filter in the passband, while the

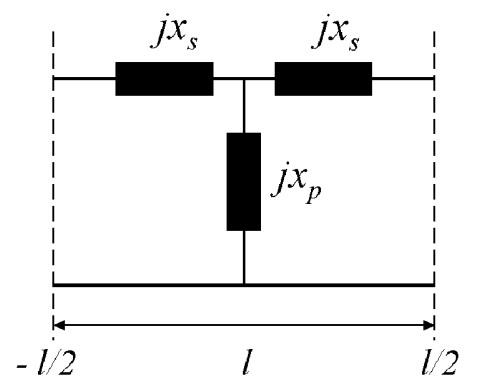

(a)

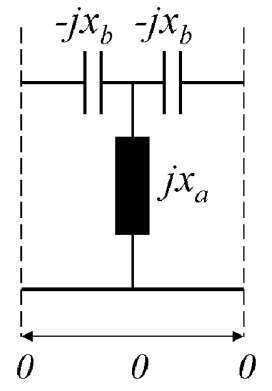

(b)
Fig. 5. Two equivalent lumped element circuits for an iris in a rectangular waveguide (a) edge representation, (b) center representation.

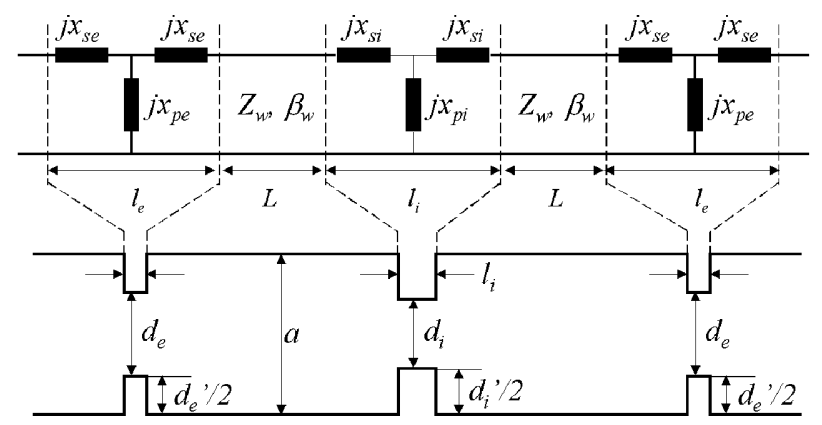

Fig. 6. Equivalent network of the filter consisting of T-sections of reactances for the irises and transmission lines for the waveguide resonator sections.

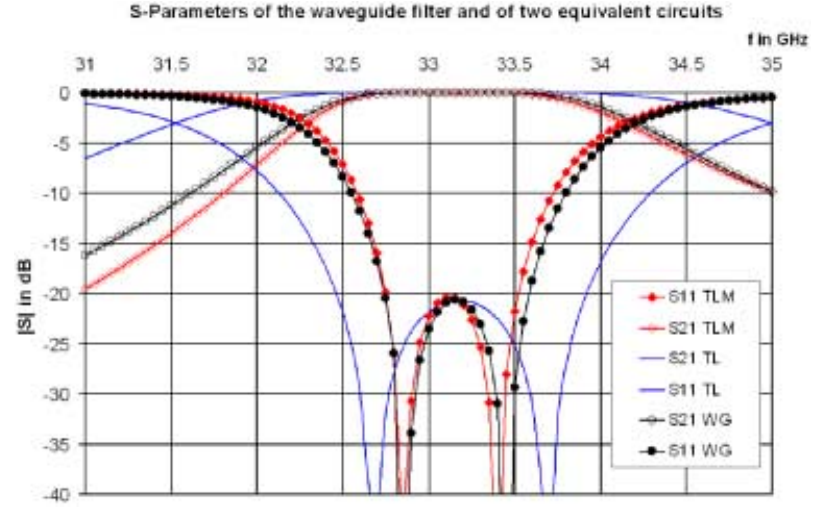

Fig. 7. S-parameters of the actual filter computed with TLM, and of two equivalent networks computed with Touchstone, one containing TEM transmission line sections (TL) and the other transmission line sections having the dispersive properties of the $\mathrm{TE}_{10}$ mode.

equivalent circuit containing non-dispersive transmission line sections has almost twice the bandwidth of the original filter. We will thus use the model with dispersive resonator sections to specify the desired filter characteristics.

The specifications of two possible equivalent circuits are given in Table 1 . They are electrically equivalent and will serve as starting points for the synthesis. The midband frequency is $33.12 \mathrm{GHz}$, and the waveguide width is $7 \mathrm{~mm}$. The cutoff angular frequency is thus $\pi c / 7 \mathrm{~mm}$.

The frequency dependence of these parameters is assumed to be as follows:

$$
\begin{aligned}
& x_{s e, i}=\frac{\omega L_{s e, i}}{Z_{w}} ; x_{p e, i}=\frac{\omega L_{p e, i}}{Z_{w}} ; Z_{w}=\frac{\eta_{0}}{\sqrt{1-\left(\omega_{c} / \omega\right)^{2}}} \\
& \text { and } \beta_{w}=\frac{\omega \sqrt{1-\left(\omega_{c} / \omega\right)^{2}}}{c}
\end{aligned}
$$

The inductances $L_{s}$ and $L_{p}$ can be considered constant over the bandwidth of the filter.

\subsection{Generation of a starting geometry}

The S-parameters of the equivalent network in Figure 6 could be realized with a number of possible topologies (various types of inductive posts or irises in a waveguide of rectangular or cylindrical cross-section) which we may select for various reasons. We opt for selecting the original topology with symmetrical inductive irises so that we can obtain dimensions similar to those used to generate our filter specifications in the first place. However, we must specify some starting values for the filter dimensions. Such values can be generated using formulas given by Marcuvitz in the Waveguide Handbook [7]. Note that the same equivalent circuit elements can be realized by irises with rectangular, elliptical or other possible profiles. The end result of the synthesis will thus depend on the 
Table 1. Data for two possible filter equivalent circuits that may be used to synthesize the bandpass filter. The two upper rows specify a circuit with irises represented as in Figures $5 \mathrm{a}$ and 6, while the two lower rows specify a circuit with irises represented as in Figure 5b.

\begin{tabular}{cccccccc}
\hline$f_{0} / \mathrm{GHZ}$ & $x_{s e}$ & $x_{p e}$ & $x_{s i}$ & $x_{p i}$ & $Z_{w} / \Omega$ & $\beta_{w} / \mathrm{rad} / \mathrm{mm}$ & $L / \mathrm{mm}$ \\
\hline 33.12 & 0.21896 & 0.43266 & 0.17878 & 0.11835 & 494.174 & 0.5295429 & 4.4 \\
\hline$f_{0} / \mathrm{GHZ}$ & $x_{b e}$ & $x_{a e}$ & $x_{b i}$ & $x_{a i}$ & $Z_{w} / \Omega$ & $\beta_{w} / \mathrm{rad} / \mathrm{mm}$ & $L / \mathrm{mm}$ \\
\hline 33.12 & 0.10253 & 0.32969 & 0.19623 & 0.10967 & 494.174 & 0.5295429 & 5.7 \\
\hline
\end{tabular}

starting geometry we select, owing to the non-uniqueness of the inverse process.

It is common practice in waveguide bandpass design to select the thickness of the septa at the start, and we do the same here to expedite the task, setting $l_{e}=1.2 \mathrm{~mm}$ and $l_{t}=1.4 \mathrm{~mm}$ as in Figure 4. The starting values for the iris widths $d_{e}$ and $d_{i}$ can be found using the following approximate formula:

$$
d \approx \frac{2 a}{\pi} \tan ^{-1} \sqrt{\frac{\left(x_{s}+2 x_{p}\right) \lambda_{g}}{a}}
$$

Entering the values for the equivalent discontinuities given in Table 1 yields the following approximate dimensions:

$$
d_{e} \approx 4.1723 \mathrm{~mm} ; \quad d_{i} \approx 3.1159 \mathrm{~mm} .
$$

We select even larger values $\left(d_{e}=4.2 \mathrm{~mm}\right.$ and $d_{i}=$ $3.4 \mathrm{~mm}$ ) to demonstrate the convergence of the synthesis procedure. The starting geometry for the synthesis of the iris contours is thus given in Figure 8.

\subsection{Identification of the designable boundary parts}

In accordance with usual practice we will fix the width $a$ of the waveguide as well as the length of the resonators which are well defined in the specifications. The apertures of the irises are thus the only dimensions that will be modified to achieve the desired characteristics. Hence, the designable boundary parts are the end faces of the septa. Since the filter is symmetrical with respect to its longitudinal axis, only half of the structure needs to be modeled.

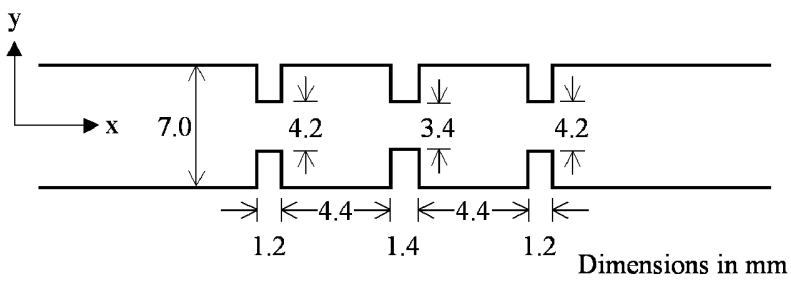

Fig. 8. Starting geometry for the filter synthesis. In this example, the width of the irises and the distance between them have been kept the same as in the original filter, but the iris openings are 0.4 $\mathrm{mm}$ wider than the original dimensions.

\subsection{Determination of the characteristic frequencies}

Characteristic frequencies are resonant frequencies of the filter that are determined by the position of the designable boundaries. Resonances are created by the strategic insertion of electric and magnetic walls. Consider the split equivalent T-circuit of the irises (Figure 9) when its two ports are subject to even and odd excitation. If we connect sections of short-circuited waveguides of width "a" to the even and odd sub-circuits of the iris, we create resonators subject to the following resonance conditions:

Even case: $x_{s}+2 x_{p} \tan \beta L_{e}=0 \Rightarrow L_{e}=\frac{\tan ^{-1}\left(-2 x_{p}-x_{s}\right)}{\beta}$

Odd case: $x_{s}+\tan \beta L_{0}=0 \quad \Rightarrow \quad L_{0}=\frac{\tan ^{-1}\left(x_{s}\right)}{\beta}$

Since we know the normalized inductances and the characteristics of the waveguide at mid-band frequency of the filter (Table 1 and Eq. 1), we can determine the lengths $L_{e}$ and $L_{o}$. Both resonant structures have thus the same resonant frequency, namely the mid-band frequency $f_{0}=$ $33.12 \mathrm{GHz}$, and we have thus determined a characteristic even and a characteristic odd resonant frequency for each iris. The next step is to realize these resonating structures, discretize them using the Generalized Symmetrical Condensed Node (GSCN) TLM model, and use them to synthesize the designable boundary of the irises. The even and odd resonance conditions are shown in Figure 10.

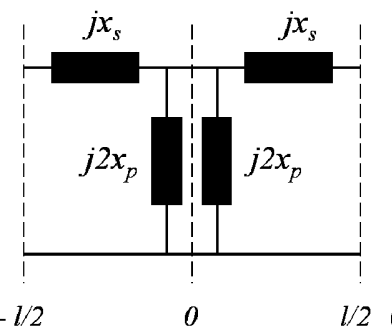

(a)

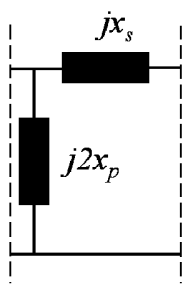

$l / 2 \quad 0$

(b)

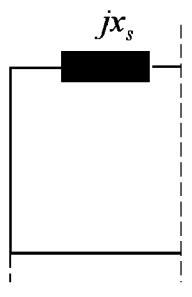

(c)
Fig. 9. Equivalent T-Circuit of a thick symmetrical waveguide iris (a) and representation of its port impedance in the cases of even (b) and odd (c) excitation of its ports. 

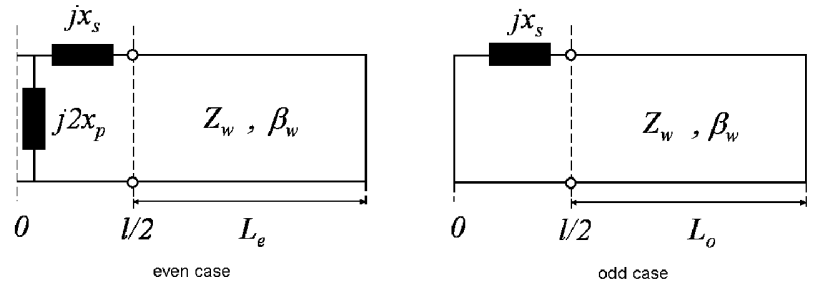

Fig. 10. Resonators formed by connecting short-circuited waveguide sections to the even and odd equivalent circuits of the iris, with resonance conditions given by Eqs. 3 and 4.

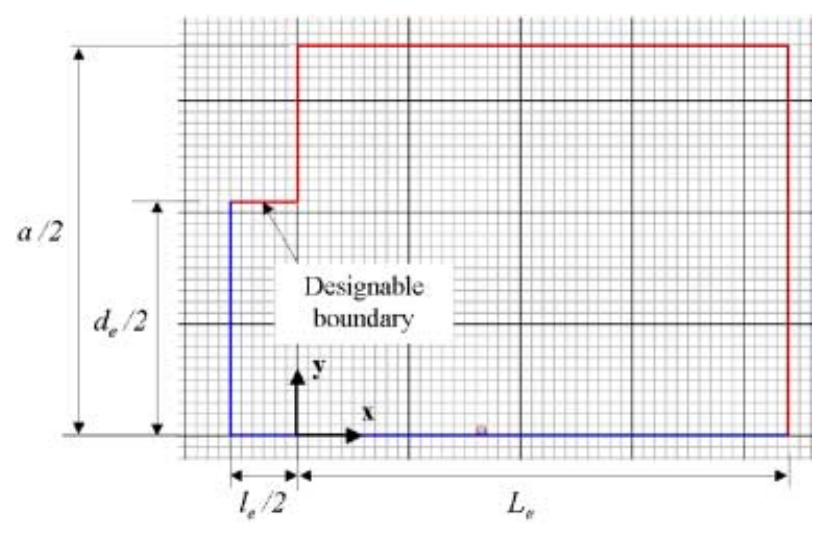

Fig. 11. Starting geometry of the resonant structure for the even characteristic frequency, containing the external iris. The grid size is $0.1 \mathrm{~mm}$.

\subsection{Monochromatic field injection}

Figure 11 shows the even mode resonator containing the external iris. The length $L_{e}$ has been computed with Eq. 3 at mid-band frequency, and $d_{e}$ is the initial guess position of the designable boundary determined with Eq. 2 and rounded to $4.2 \mathrm{~mm}$. Since the value of $d_{e}$ is not correct, the natural resonant frequency will be different from the midband frequency $f_{0}$. However, if the structure does resonate at its natural frequency, the field distribution along the designable boundary will be similar to that in the structure with the correct dimensions. We therefore perform a short analysis of the first guess structure, record and store the spatial profile of incident TLM pulse amplitudes along the designable boundary, then replace the latter by an array of matched monochromatic TLM sources, and finally inject sampled monochromatic signals with the recorded profile at the desired resonant frequency $f_{0}$. This procedure has been implemented in the Field Simulator MEFiSTo3D Pro ${ }^{\mathrm{TM}}$. A steady-state field builds up as shown in Figure 12.

\subsection{Extraction of optimal boundary geometry and validation of results}

Visual inspection of the false color field plot in Figure 12 clearly indicates that the contour of the iris septum must

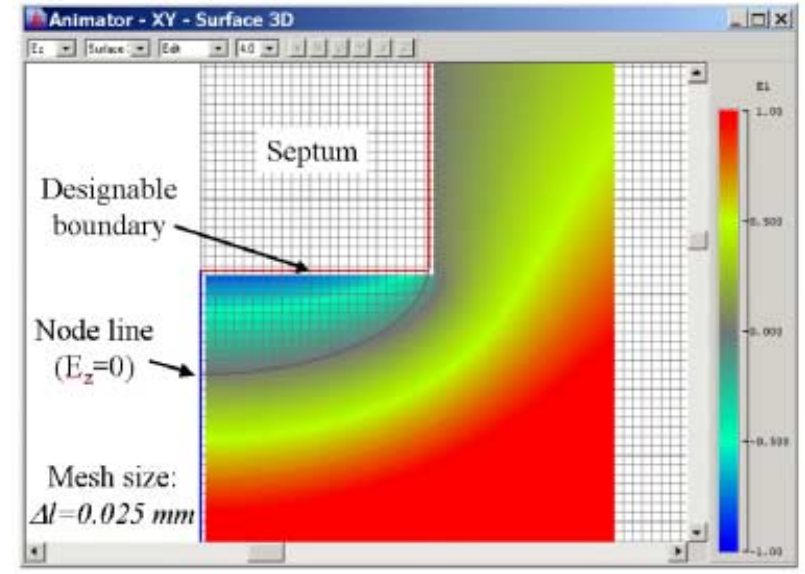

Fig. 12. Standing field pattern in the vicinity of the external iris when a monochromatic signal at $33.12 \mathrm{GHz}$ is injected from the designable boundary. The node line $\left(E_{z}=0\right)$ suggests the optimal contour of the front of the iris septum for a resonant frequency of $33.12 \mathrm{GHz}$.

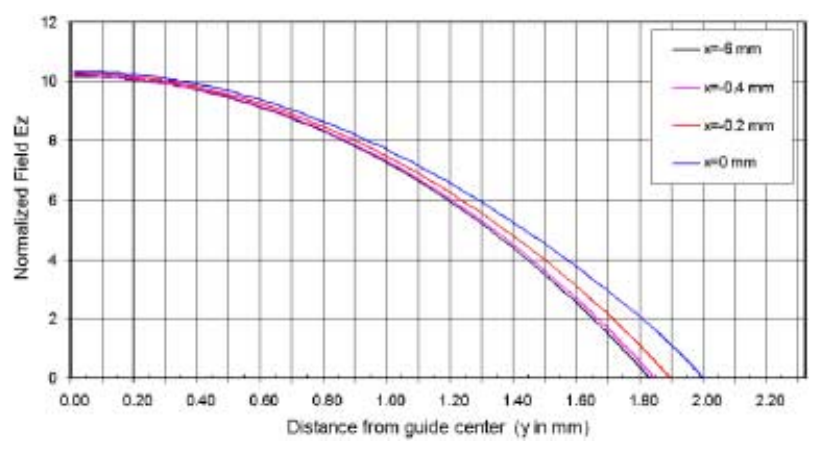

Fig. 13. Electric field along lines parallel to the y-axis (see Fig. 11 for coordinate definition). The optimized septum should be located where these lines go through zero.

be elliptical for the structure to resonate at $33.12 \mathrm{GHz}$. A more accurate definition of this contour is found by drawing the field profile along lines parallel to the y-axis and determining the loci at which $E_{z}=0$, as shown in Figure 13. The field data can be exported from the Field Simulator MEFiSTo-3D Pro ${ }^{\mathrm{TM}}$ to a text file and then processed with EXCEL or more advanced data processing tools.

Only two field simulations (one for the external and one for the internal iris geometry) are necessary to reach the optimized contour topology, even though the initial guess is off by typically ten percent. This does not include the short initial simulation to determine the amplitude and phase distribution along the designable boundary. Figure 14 shows the initial rectangular iris profiles from which the specifications have been developed (profile a), and compares them to the first guess profile (profile b) and the synthesized elliptical profile (profile c). The latter profile was implemented in the form of elliptic electric boundaries that were fitted to the shape of the node line extracted from the stationary field pattern after monochro- 


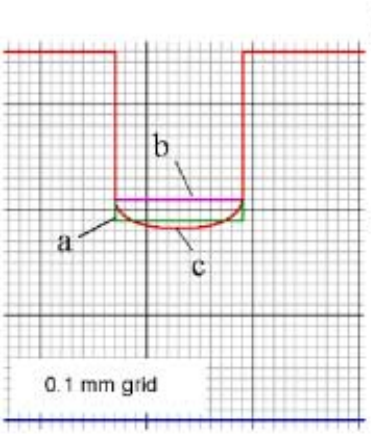

Profile of external iris

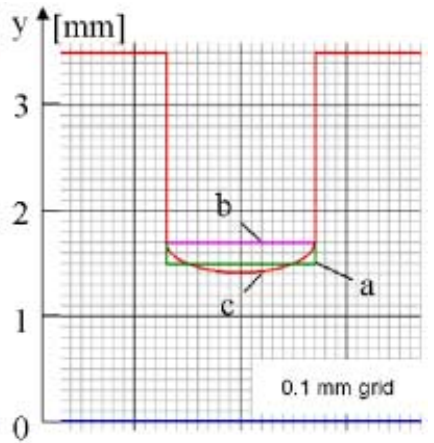

Profile of internal iris
Fig. 14. Comparison of iris profiles before and after synthesis. The three contours are: (a) Original rectangular contour from which specifications were derived (Figure 4); (b) Starting geometry computed with the approximate expression 2 (Figure 8); (c) Optimal elliptical contour synthesized by monochromatic injection from contour b.

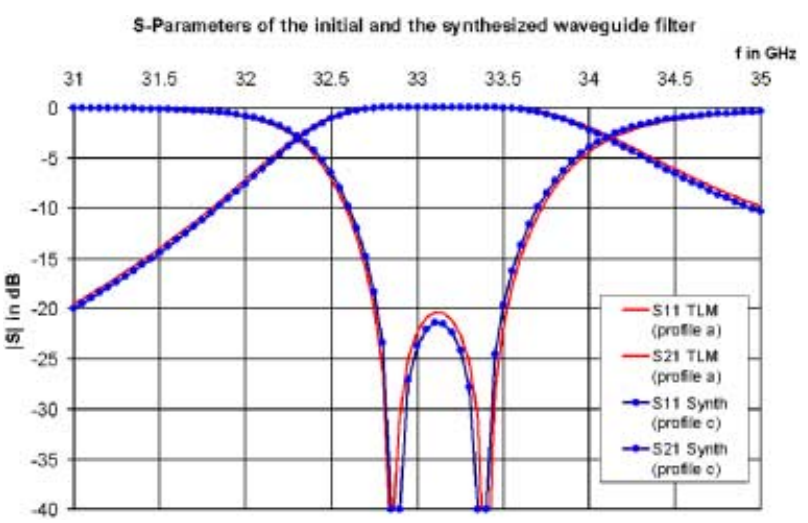

Fig. 15. S-parameters of the initial waveguide filter (profile a) and the optimal synthesized filter (profile c) obtained by numerical analysis with TLM. Excellent agreement is achieved.

matic injection. To validate the specifications of the synthesized filter, Figure 15 compares the S-parameters of the filters with the profiles a and c. The agreement between the two sets of S-parameters is well within the resolution capability of the numerical modeling technique and can be qualified as excellent, given the very high sensitivity of the $\mathrm{S}$-parameters to the dimensions of the irises. In fact, the synthesis of high-Q structures such as filters is particularly well suited to demonstrate the accuracy of field-based synthesis.

\section{Conclusions}

We have demonstrated the method of field-based synthesis of electromagnetic structures using monochromatic field injection. We have applied this new approach to the electromagnetic design of a waveguide bandpass filter with the objective to demonstrate the accuracy and effectiveness of this alternative design methodology. Using the Transmission-Line Matrix (TLM) modeling method for time-domain simulation of electromagnetic fields we have generated optimal boundary topologies by means of stationary field pattern that minimize energy losses, maximize power-handling capability and reduce sensitivity to dimensional tolerances. After a theoretical introduction to the synthesis methodology we have presented a step-by-step synthesis of an iris-coupled rectangular waveguide bandpass filter and verified the result by performing a rigorous field analysis of the synthesized topology. The agreement between the original specifications and the S-parameters of the synthesized filter is excellent and demonstrates the high degree of accuracy that can be achieved with this approach in a single field simulation per designable parameter. The field synthesis approach thus combines remarkable accuracy with high numerical efficiency.

\section{References}

[1] Bakr, M.H.; Hoefer, W.J.R.: Electromagnetic synthesis: A novel concept in microwave design. In: 21st European Microwave Conference Dig., London, UK, 2001.

[2] So, P.P.M.; Bakr, M.H.; Hoefer, W.J.R.: A paradigm for electromagnetic field synthesis by monochromatic injection. In: 2002 ACES Symposium Digest, Monterey, CA, 2002.

[3] Bakr, M.H.; So, P.P.M.; Hoefer, W.J.R.: Time domain synthesis exploiting 3d tlm symmetric condensed nodes. In: 2002 IEEE International Microwave Symposium Dig., Seattle, WA, June 2002.

[4] Bakr, M.H.; So, P.P.M.; Hoefer, W.J.R.: The generation of optimal microwave topologies using time domain field synthesis. IEEE Trans. Microwave Theory Tech. Vol. MTT-50, No. 11 (2002), 2537-2544

[5] Matthaei, G.; Young, L.; Jones, E.M.T.: Microwave filters, impedance-matching networks, and coupling structures. Artech House Books, Norwood 1980.

[6] Amari, S.; Rosenberg, U.; Bornemann, J.: Adaptive synthesis and design of resonator filters with source/load-multiresonator coupling. IEEE Trans. Microwave Theory Tech. MTT-50, No. 8 (2002), 1969-1978.

[7] Marcuvitz, N.: Waveguide handbook. Boston Technical Pulishers Inc., Boston 1964.

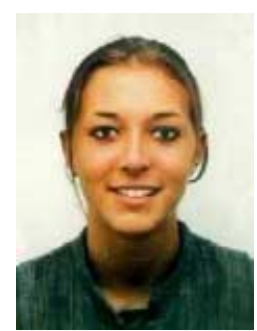

Aurélie Sallier received the engineering degree in Electronics and Communication Systems, and the Diplôme d'Etudes Approfondies (DEA) in Electronics, from the National Institute of Applied Sciences (INSA) in Rennes, France, in 2002. During her studies she participated in several research and industrial projects, in particular a four-month project involving the computer simulation of indoor transmission using ultra-wideband pulse radio technology, and a four-month testing and validation project involving the RDY2 radar system at Thales Airborne Systems in Pessac, France. From March to September 2002 she spent six months at the 
Computational Electromagnetics Research Laboratory (CERL) at the University of Victoria, Canada, where she conducted research on electromagnetic field synthesis in the time domain. This work formed the subject of her compulsory research project for her engineering degree at INSA Rennes, and for this paper as well. In September 2002 she returned to the National Institute of Applied Sciences in Rennes where she is currently studying towards her doctoral degree.

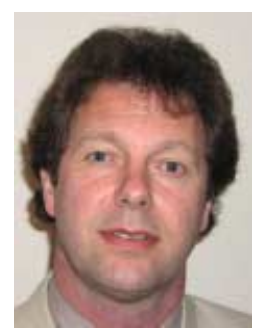

Jens Bornemann received the Dipl.-Ing. and the Dr.-Ing. degrees, both in electrical engineering, from the University of Bremen, Germany, in 1980 and 1984, respectively.

From 1984 to 1985 , he was a consulting engineer. In 1985, he joined the University of Bremen, Germany, as an Assistant Professor. Since April 1988, he has been with the Department of Electrical and Computer Engineering, University of Victoria, Victoria, B.C., Canada, where he became a Professor in 1992. From 1992 to 1995, he was a Fellow of the British Columbia Advanced Systems Institute. In 1996, he was a Visiting Scientist at Spar Aerospace Limited (now EMS Technologies Inc.), Ste-Anne-de-Bellevue, Québec, Canada, and a Visiting Professor at the Microwave Department, University of Ulm, Germany. From 1997 to 2002, he was a co-director of the Center for Advanced Materials and Related Technology (CAMTEC), University of Victoria. From 1999 to 2002, he served as an Associate Editor of the IEEE Transactions on Microwave Theory and Techniques in the area of Microwave Modeling and CAD. $\mathrm{He}$ has coauthored Waveguide Components for Antenna Feed Systems. Theory and Design (Artech House, 1993) and has authored/coauthored more than 180 technical papers. His research activities include RF/wireless/microwave/millimeter-wave components and systems design, and problems involving electromagnetic field theory in integrated circuits, feed networks and radiating structures.

Dr. Bornemann is a Registered Professional Engineer in the Province of British Columbia, Canada. He is a Fellow IEEE and serves on the Technical Program Committee of the IEEE MTT-S International Microwave Symposium and the editorial advisory board of the International Journal of Numerical Modelling.

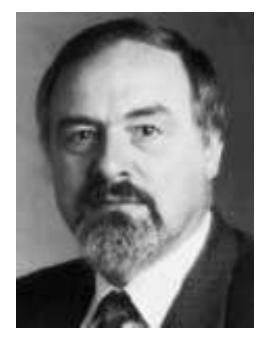

Wolfgang J.R. Hoefer received the Dipl.-Ing. degree in Electrical Engineering from the Technische Hochschule Aachen, Germany, in 1965, and the D. Ing. degree from the University of Grenoble, France, in 1968.

From 1968 to 1969 , he was a Lecturer at the Institut Universitaire de Technologie de Grenoble and a Research Fellow at the Institut National Polytechnique de Grenoble, France. In 1969 he joined the Department of Electrical Engineering, the University of Ottawa, Canada, where he was a Professor until March 1992. Since April 1992 he holds the NSERC Industrial Research Chair in Radio Frequency Engineering in the Department of Electrical and Computer Engineering, the University of Victoria, Canada. During several sabbatical leaves he has been Visiting Scientist and Professor at the following establishments: the Space Division of AEG-Telefunken in Backnang, Germany (now ATN), the Electromagnetics Laboratory of the Institut National Polytechnique de Grenoble, France, the Space Electronics Directorate of the Communications Research Centre in Ottawa, Canada, the University of Rome "Tor Vergata", Italy, the University of Nice - Sophia Antipolis, France, the Technical University of Munich, the Ferdinand Braun Institute for High Frequencies, Berlin, and the Gerhard Mercator University in Duisburg, Germany.

His research interests include numerical techniques for modeling electromagnetic fields and waves, computer aided design of microwave and millimeter wave circuits, microwave measurement techniques, and engineering education. He serves regularly on the Technical Program Committees of IEEE-MTT and AP Symposia, is the Chair of the MTT Technical Committee on Field Theory (MTT-15), co-founder and Managing Editor of the International Journal of Numerical Modelling, served as Associate Editor of the IEEE-MTT Transactions from 1998 to 2000, and as co-editor of the 2002 IEEE MTT Special December Issue. He serves on the editorial boards of the IEEE-MTT Transactions, Proceedings of the IEE, the International Journal of Microwave and Millimeter-Wave Computer Aided Engineering, Electromagnetics, and the Microwave and Optical Technology Letters. He was an "Invited Lansdowne Lecturer" of the University of Victoria, B.C., in 1989, and was awarded the "Peter B. Johns Prize" for the best paper published in the International Journal of Numerical Modelling in 1990. He was elected Fellow of the IEEE for "contributions to the modeling and design of passive microwave and millimeter-wave circuits" in 1991, and Fellow of the British Columbia Advanced Systems Institute (BC-ASI) in 1992. 